\title{
Quantitative Comparison of Protein Surface Cov- erage on Glass Slides and Silver Island Films in Metal-Enhanced Fluorescence-based Biosensing Applications
}

\author{
Tsehai A.J. Grell, ${ }^{1}$ Eduardo Paredes, ${ }^{2}$ Subha R. Das,${ }^{2}$ and Kadir Aslan ${ }^{*} 1$ \\ ${ }^{1}$ Morgan State University, Department of Chemistry, 1700 East Cold Spring Lane, Baltimore, MD, 21251, USA. \\ ${ }^{2}$ Carnegie Mellon University, Department of Chemistry, 4400 Fifth Avenue Pittsburgh, PA 15213, USA \\ *Corresponding authors. Email: Kadir.Aslan@morgan.edu
}

\begin{abstract}
The use of Metal-Enhanced Fluorescence (MEF) phenomenon in fluorescence-based bioassays affords for increased sensitivity to be realized by incorporating metal nanoparticles onto planar surfaces. The close-range interactions of metal-fluorophores result in increased fluorescence emission from the bioassays, which in turn affords for the detection of target biomolecules at lower concentrations. Moreover, the use of silver nanoparticles increases the photostability of fluorophores improving the detectability of fluorescence emission under prolonged use of excitation light. Although numerous reports on MEF-based biosensing applications exist, the contribution of protein coverage on Silver Island Films (SIFs) on the increased fluorescence emission was never investigated. This work presents our findings on the quantitative comparison of protein surface coverage on SIFs and blank glass slides. In this regard, identical protein bioassay for a model protein (biotinylated bovine serum albumin, b-BSA) on these surfaces is constructed and the relative extent of protein surface coverage on SIFs and blank glass slides was determined using radio-labeled biomolecules. It was found that the total scintillation counts on SIFs and blank glass slides were similar for BSA concentrations ranging from $1 \mu \mathrm{M}$ to $1 \mathrm{pM}$, which implies that increased fluorescence in MEF-based biosensing applications is only due to metal-fluorophore interactions.
\end{abstract}

Keywords: Metal-enhanced fluorescence; Plasmon-controlled fluorescence; Silver island films; Gold nanoparticles; Quenching

Citation: T.A.J. Grell et al. Quantitative Comparison of Protein Surface Coverage on Glass Slides and Silver Island Films in Metal-Enhanced Fluorescence-based Biosensing Applications. Nano Biomed Eng. 2010, 2(3), 165-170. DOI: 10.5101/nbe.v2i3.p165-170.

\section{Introduction}

Metal-Enhanced Fluorescence (MEF) is a phenomenon, which describes the close-range interactions between plasmon resonant metal particles and fluorophores. The metal-fluorophore interactions result in 1) non-radiative transfer of energy from the excited state of the fluorophores to surface plasmons of the metal nanoparticles (this energy is re-radiated as fluorescence emission at the same wavelength by the nanoparticles into the free-space), 2) in enhanced absorption of light by fluorophores due to the increased electric fields between and around the metal nanoparticles, and 3) improved photostability of fluorophores. The size and type of the metal nanoparticles and the wavelength of emission of fluorophores are the main factors affecting the efficiency of the metal particle-fluorophore interactions. It is well-known that the extinction spectrum for metal nanoparticles $<40 \mathrm{~nm}$ and $>40 \mathrm{~nm}$ in size is dominated by absorption and scattering, respectively [1]. Since MEF phenomenon is related to absorption and scattering components of the metal nanoparticles, 
while metal nanoparticles $<40 \mathrm{~nm}$ in size were shown to quench fluorescence [2], enhanced fluorescence emission is observed when metal nanoparticles $>40$ $\mathrm{nm}$ in size are used [3, 4]. In addition, the most efficient non-radiative energy transfer from the fluorophores at their excited states to surface plasmons occur when the wavelength of fluorescence emission and surface resonance peak of metal nanoparticles overlap [2].

The benefits of MEF phenomenon described above lend itself to the incorporation of metal nanoparticles into fluorescence-based biosensing applications, such as immunoassays [5] and fluorescence in-situ hybridization assays [6]. In the current fluorescence-based biosensing applications, typically fluorophores with high-quantum yields are employed to increase the sensitivity of the bioassays, which results in high background emission and poor fluorophore photostability. The use of metal nanoparticles (such as silver [7], gold [8], copper [9], aluminum [10]) in these applications afford for the use of low-quantum yield fluorophores, which can withstand the prolonged exposure to excitation light for multiple measurements to be done. In this regard, numerous reports on MEF-based biosensing applications can be found in literature [11-15].

During the peer-review of manuscripts and scientific presentations related to MEF phenomenon and MEFbased biosensing applications, the question "How does the surface coverage of fluorophores (in fundamental MEF studies) or proteins (in MEF-based biosensing applications) on Silver Island Films (SIFs) and blank glass slides compare?” is always asked. This question arises from the fact that metal nanoparticles have 3-D structure as compared to 2-D structure of the planar surfaces such as glass slides. Subsequently, one has to consider whether the deposition of silver nanoparticles on planar slides change the surface area available for fluorophore or protein binding. Since the fluorescence emission from SIFs is significantly larger than those from blank glass slides, the contribution of fluorophore / protein coverage to the increased fluorescence emission has to be determined for accurate evaluation of metal nanoparticle-deposited surfaces for MEF applications. Despite the presence of numerous publications on MEF phenomenon [16-18] and MEF-based applications [15, 19, 20], the quantitative comparison of fluorophore or protein surface coverage on SIFs and blank glass slides was never investigated.

In this work, we present the results of our investigation of protein coverage on SIFs and blank glass slides. To quantitatively compare the protein coverage on these surfaces, identical protein bioassay for a model protein (biotinylated bovine serum albumin, b-BSA) is constructed, similar to the MEF-based bioassays previously reported [21]. The quantitative comparison of protein coverage can only be made with labeling molecules that do not interact with silver nanoparticles. In this regard, a radio-labeled detector biomolecule (ra- dio-labeled-biotinylated oligonucleotide) is employed instead of a typical fluorophore-labeled biomolecule used in MEF-based bioassays. Optical absorption spectroscopy and scanning electron microscopy techniques were employed to characterize SIFs. After the construction of the bioassay on SIFs and blank glass slides, the total scintillation counts from both surfaces were measured and compared. It was found that the total scintillation counts on SIFs and glass slides were similar for b-BSA concentrations ranging from $1 \mu \mathrm{M}$ to 1 $\mathrm{pM}$. These observations imply that the increased fluorescence emission from SIFs (and surfaces with other metal nanoparticles) in MEF-based biosensing applications is due to the metal-fluorophore interactions without any contribution from changes in protein surface coverage. These results also apply to low affinity biological interactions, such as antigen-antibody interactions used in immunoassays.

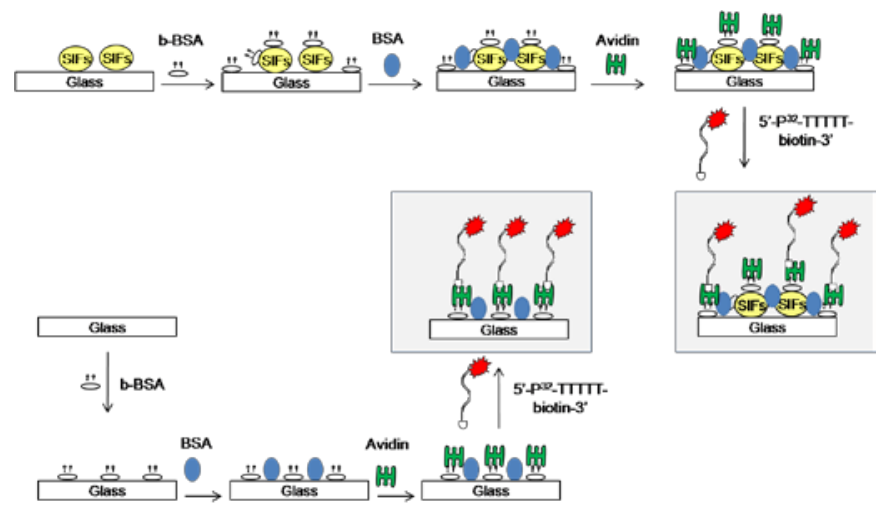

Figure 1. Schematic depiction of the protein bioassay for a model protein (b-BSA) on silver island films (SIFs) and blank glass slides.

\section{Materials and methods}

Materials. 3'-biotin Controlled Pore Glass columns, phosphoramidites with labile pheoxyacetyl (PAC) protecting groups and appropriate reagents for solid phase synthesis of DNA were purchased from ChemGenes or Glen Research. Silver nitrate (>\%99), D-glucose, ammonium hydroxide, sodium hydroxide, biotinamidocaproyl-labeled bovine serum albumin (b-BSA), BSA, avidin, Silane-prep glass microscope slides were purchased from Sigma-Aldrich Chemical Co. (Milwaukee, WI).

Nucleic acid synthesis and preparation. Solid phase oligonucleotide synthesis was performed on a MerMade 4 instrument (Bioautomation). Synthesis and deprotection of 3'-biotinylated-T5 (5'-TTTTT-biotin-3') was conducted using standard protocols for PAC protected amidites as recommended by the manufacturer. 5'-end radiolabeling was conducted with T4 PNK using $\gamma$-32P ATP using standard protocols. Radiolabeled 3'biotinylated-T5 (5'-[32P]-TTTTT-biotin-3') was purified by electrophoresis on $8 \mathrm{M}$ urea polyacrylamide gels. The DNA band was excised and eluted overnight at 
$4^{\circ} \mathrm{C}$ in $10 \mathrm{mM}$ Tris-0.1 mM EDTA buffer (pH 7.5) and desalted on a Waters C18 Sep-Pak column.

Deposition of Silver Island Films (SIFs). SIFs were deposited onto silanized glass slides using Tollen's reaction scheme. In this regard, first a solution of silver nitrate $(0.5 \mathrm{~g}$ in $60 \mathrm{ml}$ of deionized water) was placed in a clean $100-\mathrm{ml}$ glass beaker. While stirring at the quickest speed (on a Corning Heater/Stirrer), $200 \mu \mathrm{L}$ of freshly prepared $5 \%(\mathrm{w} / \mathrm{v})$ sodium hydroxide solution is added, which results in the formation of dark brown precipitates of silver particles. The precipitates were redissolved by $2 \mathrm{ml}$ ammonium hydroxide. The resultant clear solution is cooled down to $5^{\circ} \mathrm{C}$ by placing the beaker in an ice bath, followed by soaking the silanized glass slides in the solution. After $2 \mathrm{~min}$, a fresh solution of D-glucose ( $0.72 \mathrm{~g}$ in $15 \mathrm{ml}$ of water) is added. Subsequently, the temperature of the mixture is then warmed to $30^{\circ} \mathrm{C}$. As the color of the mixture turns from "yellow-green" to "yellow-brown" and the color of the slides become green (in $2 \mathrm{~min}$ ), the slides are removed from the mixture. SIFs were washed with water and sonicated for 30 seconds at room temperature. SIFs were then rinsed with deionized (DI) water several times and air-dried. A cross-section $(12 \times 40 \mathrm{~mm} 2)$ of the blank glass slides and SIFs were cut and kept in DI water until further use.
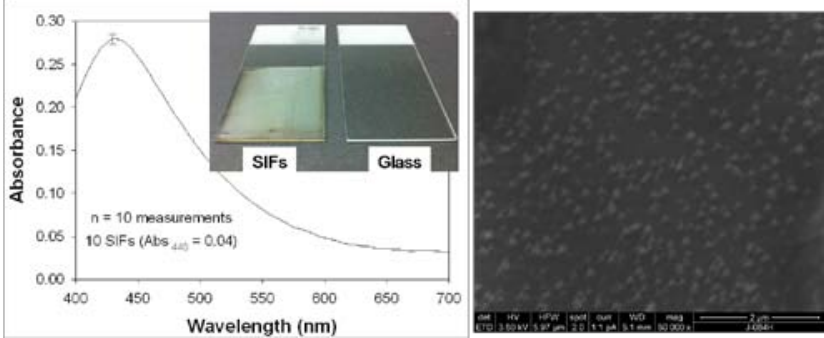

Figure 2. Left- Optical absorption spectrum of 10 different SIFs. Left-Inset: Real-color photograph of SIFs and blank glass slide slides. Right- Scanning Electron Microscope image of SIFs.

All glassware used was treated with "piranha solution” (3:7 v/v; 30\% hydrogen peroxide/concentrated sulfuric acid: CAUTION! piranha solution reacts violently with most organic materials and should be handled with extreme care) and rinsed with deionized water at least three times before use.

Construction of model protein assay on SIFs and blank glass slides. In order to quantitatively compare the protein surface coverage on SIFs and blank glass slides, identical bioassay for a model protein (b-BSA) was constructed on SIFs and blank glass slides (without silver nanoparticles). Figure 1 shows the schematic depiction of the bioassay for model protein b-BSA. In the first step, a solution of b-BSA with a range of bulk concentrations ( $1 \mu \mathrm{M}$ to $1 \mathrm{pM})$ was incubated on SIFs and blank glass slides at room temperature for $20 \mathrm{~min}$. Unbound b-BSA was removed by rinsing the surfaces with deionized water several times. The surfaces were then treated with $5 \%(\mathrm{w} / \mathrm{v}) \mathrm{BSA}$ for 20 min to reduce the non-specific binding of avidin. Unbound BSA was removed by rinsing the surfaces with deionized water several times. In the next step, a $1 \mathrm{mg} / \mathrm{ml}$ solution of avidin was incubated on the surfaces for $20 \mathrm{~min}$. Unbound avidin was removed by rinsing the surfaces with deionized water several times. Subsequently, the surfaces were coated with $100 \mu \mathrm{L}$ of a $1 \mu \mathrm{M}$ solution of 3'biotinylated-T5 ( 0.015\% 5'-32P labeled) in autoclaved, deionized $(18.2 \mathrm{M} \Omega)$ water with a $1.65 \mathrm{~mL}$ pipette tip. Samples were kept at room temperature for $20 \mathrm{~min}$ to allow for biotin binding prior to rinsing. The glass slides were washed three times with $1 \mathrm{~mL}$ aliquots of water. The glass slides were placed on liquid scintillation vials to which $\sim 5 \mathrm{ml}$ of scintillation solution was added. Scintillation measurements were conducted on a LS 6500 Beckman Coulter liquid scintillation counter in triplicate. Each sample slide was done in duplicate.

Scanning Electron Microscope (SEM) images of SIFs were obtained at the Core Imaging Facility of the University of Maryland Dental School. Real-color photographs of SIFs and blank glass slides were taken using a 5 mega-pixel digital camera.

\section{Results and discussions}

SIFs are routinely used in MEF-based biosensing applications $[15,19,20]$. In this regard, the homogeneous deposition of SIFs onto planar substrates is crucial for quantitative determination of target biomolecules. Glass microscope slides are the main choice in the preparation of SIFs due to their amenability to surface modification procedures (e.g. silanization). Typically, amine-terminated silanes are employed in the silanization of glass slides based on the affinity of silver nanoparticles towards amine groups [21]. Subsequently, in this work, SIFs are deposited onto silanized glass slides presenting amine groups. Figure 2-Left shows the average absorption spectra of 10 different SIFs. The surface plasmon resonance (SPR) peak for SIFs appears at 440 , which is consistent with the literature [7]. The coefficient of variance for absorbance is calculated to be around $2 \%$ for SIFs, demonstrating the homogeneous nature of the deposition procedure in our hands. Figure 1-Left-Inset shows a real-color photograph of SIFs and blank glass slides. After the deposition of SIFs, the color glass slides were changed to the color of SIFs (green-brown). Moreover, SIFs visually appears homogeneous, which is consistent with the absorption spectrum of SIFs. This observation is also corroborated by SEM image (Figure 2-Right), which show the SIFs are deposited in a homogeneous manner. The SEM image in Figure 2-Right also reveals that the size of the silver nanoparticles is $\sim 150 \mathrm{~nm}$. The choice of the size of silver nanoparticles is based on the previous observations on MEF where the use of larger metal nanoparticles is recommended for MEF-based applications [22, 23]. 
the bioassay used in this study and those constructed for MEF-based bioassays is the type of label used for the detector biomolecule (avidin). That is, in this study a radio-labeled detector biomolecule (radio-labeledbiotinylated oligonucleotide) is used instead of a fluorophore [7].

Figure 3 shows the relative total scintillation count (in arbitrary units) measured from the identical bioassay for b-BSA constructed on SIFs and blank glass slides. As expected, the scintillation count from both surfaces is the largest for the largest concentration of bBSA $(1 \mu \mathrm{M})$ used. As the concentration of b-BSA is decreased from $1 \mu \mathrm{M}$ to $1 \mathrm{nM}$ the total scintillation count is decreased and leveled off below $0.1 \mathrm{nM}$. In addition, a control experiment (No b-BSA), where bBSA is omitted from the surface is also carried out to determine the non-specific binding of detector biomolecule. The total scintillation counts from the control experiment run on both SIFs and blank glass slides are well below those measured from the lowest concentration of b-BSA $(1 \mathrm{pM})$ in the bioassay. These results imply that the lower detection limit of b-BSA concentration is $0.1 \mathrm{nM}$ using a radio-labeled detector biomolecules. Similar lower-detection limits using fluorescence-based detection were also reported [20]. However, most research groups employ fluorescence-based detection over radio-labeled based detection due to the availability of wide-range of fluorescence instruments and the relative simplicity of fluorescence measurements.

It is important to further comment on the implications of the total scintillation counts measured from a protein bioassay constructed on SIFs and blank glass slides on MEF-based biosensing applications. As can be seen from Figure 3, the total scintillation counts for all concentrations of b-BSA are similar on SIFs and blank glass slides. That is, the extent of protein coverage on SIFs and blank glass slides are similar. To the best of our knowledge, this observation is made for the first time and provides a direct proof for the benefits of MEF phenomenon in biosensing applications. The incorporation of silver nanoparticles into bioassays run on planar surfaces does not increase the surface coverage of proteins and the observed increase in fluorescence-emission and improved photostability of fluorophores is indeed due to MEF phenomenon.

It is also important to comment on the implications of these results on low affinity biological interactions, such as antigen-antibody interactions used in immunoassays. SIFs were used in direct immunoassays [25], where the target protein is allowed to attach to the surfaces first, similar to b-BSA in this study. The target protein attaches to the silver nanoparticles through their primary amine groups. In sandwich-type immunoassays, other proteins such as protein A (or G) is used to anchor the capture antibody to the assay surface. Protein A (or G) attaches to the surfaces (SIFs [26] and 
glass slides [26]) through their primary amine groups. Subsequently, the observations made in this study directly apply to immunoassays.

\section{Conclusions}

In this work, we investigated the quantitative comparison of protein surface coverage on SIFs and blank glass slides employed in MEF-based bioassays. In this regard, SIFs were deposited onto amine-functionalized glass slides using Tollen's reaction scheme. Optical absorption spectroscopy and scanning electron microscopy revealed that SIFs were deposited onto glass slides in a homogeneous manner. SIFs displayed a surface plasmon resonance peak at $440 \mathrm{~nm}$ and the size of the silver nanoparticles was $\sim 150 \mathrm{~nm}$. To assess and compare the proteins surface coverage on SIFs and blank slides, identical protein assay for a model protein (b-BSA) was constructed on these surfaces. The concentration of b-BSA in solution was varied between 1 $\mu \mathrm{M}$ to $1 \mathrm{pM}$. The detection of b-BSA on surfaces was carried out using avidin and biotinylated-radio-labeledoligonucleotide. The total scintillation counts from the bioassays constructed on SIFs and blank glass slides were similar, which implied that the protein surface coverage on these surfaces were similar. Subsequently, it is concluded that the observed increase in fluorescence-emission and improved photostability of fluorophores in MEF-based biosensing applications is not as a result of differences in protein coverage but indeed is due to metal-fluorophore interactions.

\section{Acknowledgement}

This project was financially supported by Award Number 5-K25EB007565-04 from the National Institute of Biomedical Imaging and Bioengineering (to KA). TAJG and KA acknowledges the partial salary support by MSU. SRD acknowledges Department of Chemistry (CMU) start-up funds.

\section{References}

1. Yguerabide J, Yguerabide EE. Light-scattering submicroscopic particles as highly fluorescent analogs and their use as tracer labels in clinical and biological applications - I. Theory. Analytical Biochemistry 1998; 262: 137-156. do i:10.1006/abio.1998.2759

2. Aslan K, Perez-Luna VH. Quenched emission of fluorescence by ligand functionalized gold nanoparticles. Journal of Fluorescence 2004; 14: 401-405. doi:10.1023/B:JOF L.0000031821.74706.ea

3. Goldys EM, Drozdowicz-Tomsia K, Xie F, Shtoyko T, Matveeva E, Gryczynski I, Gryczynski Z. Fluorescence amplification by electrochemically deposited silver nanowires with fractal architecture. J Am Chem Soc 2007; 129: 12117-12122. doi:10.1021/ja071981j
4. Xie F, Baker MS, Goldys EM. Homogeneous SilverCoated Nanoparticle Substrates for Enhanced Fluorescence Detection. Journal of Physical Chemistry B 2006; 110: 23085-23091. doi:10.1021/jp062 170p

5. Van Dyke K, Van Dyke R, Luminescence Immunoassay and Molecular Applications. Boca Raton, FL.: CRC Press, 1990.

6. Difilippantonio M, Ried T, Technicolor genome analysis, in Topics in Fluorescence Spectroscopy, vol. 7, J. R. Lakowicz, Ed. New York: Kluwer Academic Publishers/Plenum Press, 2003, pp. 291-316.

7. Aslan K, Gryczynski I, Malicka J, Matveeva E, Lakowicz JR, Geddes CD. Metal-enhanced fluorescence: an emerging tool in biotechnology. Current Opinion in Biotechnology 2005; 16: 55-62. doi:10.1016/j.copbio.200 5.01.001

8. Aslan K, Malyn SN, Geddes CD. Metal-enhanced fluorescence from gold surfaces: angular dependent emission. $J$ Fluoresc 2007; 17: 7-13. doi:10.1007/s10895-006-0149-X

9. Zhang YX, Aslan K, Previte MJR, Geddes CD. Metalenhanced fluorescence from copper substrates. Applied Physics Letters 2007; 90: 173116.

10. Ray K, Szmacinski H, Lakowicz JR. Enhanced fluorescence of proteins and label-free bioassays using aluminum nanostructures. Anal Chem 2009; 81: 6049-6054. doi:10.1 $\underline{021 / \mathrm{ac} 900263 \mathrm{k}}$

11. Aslan K, Malyn SN, Geddes CD. Fast and sensitive DNA hybridization assays using microwave-accelerated metalenhanced fluorescence. Biochemical and Biophysical Research Communications 2006; 348: 612-617. doi:10.1016 j.bbrc.2006.07.093

12. Aslan K, Zhang YX, Geddes CD. Sonication-Assisted Metal-Enhanced Fluorescence-Based Bioassays. Analytical Chemistry 2009; 81: 4713-4719. doi:10.1021/ac802 $\underline{535 s}$

13. Liebermann T, Knoll W, Sluka P, Herrmann R. Complement hybridization from solution to surface-attached probe-oligonucleotides observed by surface-plasmon-fieldenhanced fluorescence spectroscopy. Colloids and Surfaces A-Physicochemical and Engineering Aspects 2000; 169: 337-350. doi:10.1016/S0927-7757(00)00449-0

14. Malicka J, Gryczynski I, Lakowicz JR. DNA hybridization assays using metal-enhanced fluorescence. Biochemical and Biophysical Research Communications 2003; 306: 213-218. doi:10.1016/S0006-291X(03)00935-5

15. Matveeva EG, Gryczynski Z, Lakowicz JR. Myoglobin immunoassay based on metal particle-enhanced fluorescence. Journal of Immunological Methods 2005; 302: 2635. doi:10.101 6/j.jim.2005.04.020

16. Wu M, Lakowicz JR, Geddes CD. Enhanced lanthanide luminescence using silver nanostructures: Opportunities for a new class of probes with exceptional spectral characteristics. Journal of Fluorescence 2005; 15: 5359. doi:10.1007/s10895-005-0213-y

17. Aslan K, Lakowicz JR, Szmacinski H, Geddes CD. Enhanced ratiometric $\mathrm{pH}$ sensing using SNAFL-2 on silver island films: Metal-enhanced fluorescence sensing. Journal of Fluorescence 2005; 15: 37-40. doi:10.1007/s108 95005-0211-0 
18. Aroca R, Kovacs GJ, Jennings CA, Loutfy RO, Vincett PS Fluorescence Enhancement from Langmuir-Blodgett Monolayers on Silver Island Films. Langmuir 1988; 4: 518521. doi:10.1021/la 00081a004

19. Aslan K, Gryczynski I, Malicka J, Lakowicz JR, Geddes CD, Metal-Enhanced Fluorescence: Application to HighThroughput Screening and Drug Discovery, in Drug Discovery Handbook, S. Gad, Ed. New Jersey: Wiley \& Sons, 2005.

20. Aslan K. Rapid Whole Blood Bioassays using MicrowaveAccelerated Metal-Enhanced Fluorescence. Nano Biomedicine and Engineering 2010; 2: 1-9.

21. Aslan K, Geddes CD. Microwave-accelerated metalenhanced fluorescence: Platform technology for ultrafast and ultrabright assays. Analytical Chemistry 2005; 77: 8057-8067. doi:10.1021/ac0516077

22. Aslan K, Malyn SN, Geddes CD. Angular-dependent metal-enhanced fluorescence from silver colloid-deposited films: opportunity for angular-ratiometric surface assays. Analyst 2007; 132: 1112-1121.doi:10.1039/b709170b

23. Aslan K, Malyn SN, Geddes CD. Angular-Dependent Metal-Enhanced Fluorescence from Silver Island Films. Chem Phys Lett 2008; 453: 222-228. doi:10.1016/j.cplet t.2008.01.034

24. Aslan K, Previte MJR, Zhang YX, Geddes CD. MetalEnhanced Fluorescence from Nanoparticulate Zinc Films. Journal of Physical Chemistry C 2008; 112: 18368-18375.
25. Aslan K, Baillie L, Geddes CD. Ultra Fast And Sensitive Detection Of Biological Threat Agents Using Microwaves, Nanoparticles And Luminescence. Journal of Medical Chemical, Biological and Radiological Defense 2010; 8: 1-21.

26. Aslan K, Geddes CD. Microwave Accelerated and Metal Enhanced Fluorescence Myoglobin Detection on Silvered Surfaces: Potential Application to Myocardial Infarction Diagnosis. Plasmonics 2006; 1: 53-59. doi:10.1007/s1146 8-006-9006-7

Received 23 Aug, 2010; accepted 22 Sep, 2010; published online 2 Oct, 2010.

Copyright: (C) 2010 T.A.G. Grell et al. This is an open access article distributed under the terms of the Creative Commons Attribution License, which permits unrestricted use, distribution, and reproduction in any medium, provided the original author and source are credited. 